\section{A DBASE III program that performs significance testing for the Kappa coefficient}

\author{
TIM S. C. CHAN \\ University of Hong Kong, Hong Kong
}

Interrater agreement on nominal data is often tested using the Kappa coefficient and weighted Kappa. Proposed by Cohen (1960), the Kappa statistic provides a natural means of correcting for chance and can be interpreted as an intraclass correlation coefficient.

Several computer programs have been developed for the determination of Kappa; three are written in BASIC (Bloor, 1983; Burns \& Cavallaro, 1982; Watkins \& Larimer, 1980) and one in Pascal (Powers, 1985). The present program differs from those mentioned above in that (1) it is written in DBASE III, which allows easy interface with data saved in the data base; (2) it reports both weighted and unweighted Kappa at the same time, as well as the standard error associated with them for further critical ratio tests; (3) it allows for more flexible input method; and (4) it allows for interrater agreement to be found between any two raters (out of a maximum of eight).

The weights used for this program are identical to those proposed by Fleiss and Cohen (1973), who showed that aside from a term involving the factor $1 / n$, the intraclass correlation coefficient is identical to weighted Kappa, provided the weights are taken as

$$
w_{i j}=1-\frac{(i-j)^{2}}{(k-1)^{2}} \text {. }
$$

Using the appropriate formulas of standard errors of Kappa and weighted Kappa developed by Fleiss (1981), two hypotheses can be tested for significance in this program: (1) that the underlying value of weighted Kappa is zero, and (2) that the value is equal to a prespecified $k_{w}$, other than zero.

Input. There are three forms of input method. First, the user can input frequencies in the form of a frequency matrix. Second, the user can choose to create a DBASE III data file (with .dbf extension) with data entered for up to eight observers and as many cases as would fit the diskette. Third, the user can read directly from a disk file, where rows represent subjects and columns represent variables with nominal values.

In the first form, users are asked to supply the number of rating points in order to draw up a frequency matrix of the correct size. In the second form, the user is asked to supply the name of the DBASE III data file (with .dbf extension assumed) as well as the number of rating categories. Since data can be entered for more than two observers, two rater numbers must be selected to obtain

The author's mailing address is: Dental Data Processing Unit, Faculty of Dentistry, University of Hong Kong, 34 Hospital Rd., Hong Kong the interrater agreement between the two raters (e.g., between 1 and 6). In the third option, the user is asked to input the name of a pre-prepared data file, with rows representing subjects and columns representing variables; the name of the variable on which the interrater agreement is to be found; the number of observers; and the name of the observer field. After the data file is converted to a recognizable $\mathrm{dbf}$ format, the user can then proceed to use Option 2 of the menu.

Output. If the user chooses to input data in a frequency matrix or in a .dbf file, the program outputs an $n \times n$ matrix of observed frequency cells. It is then followed by a display of Kappa coefficient, weighted Kappa, and percentage of agreement. The user is then given options to (1) test the Kappa value against zero, or (2) test the Kappa coefficient against some prespecified value, or (3) exit. For the first two options, the standard errors of Kappa and weighted Kappa are reported and the significance of the given Kappa or weighted Kappa is tested. For a two-tailed test at alpha $=.05$, flashing asterisks are shown beside a significant Kappa.

Language and Computer. The program is written in DBASE III (Aston-Tate, 1985) interpretive programming language and occupies about $6 \mathrm{~K}$ of memory. It was developed on an 80286-based IBM PC/AT microcomputer with 512K of RAM. It can also be run on IBM PC, IBM $\mathrm{PC} / \mathrm{XT}$, and other IBM compatibles.

Limitations. (1) The total number of raters as well as the number of rating points is limited to 8. (2) The total amount of memory required is the same as that required for running DBASE III (i.e., 256K). Faster performance can be expected by running the program on hard disk or with more memory. (3) The DBASE III data file must have a structure created with field names ranging from R1 to R8, depending on the number of raters. For existing data files with different field names, use MODIFY STRUCTURE to change the field name and append the data to a new file with the desirable field names.

Availability. Program listings and brief user's documentations are available upon request from the author. Send a formatted blank 51/4-in. diskette and postage cost for airmail delivery; the program will be copied without charge.

\section{REFERENCES}

Aston-Tate (1985). DBASE III 1.10. Culver City, CA: Author. BLOOR, R. N. (1983). A computer program to determine interrater reliability for dichotomous-ordinal rating scales. Behavior Research Methods \& Instrumentation, 15, 615.

Burns, E., \& Cavallaro, C. A. (1982). A computer program to determine interobserver reliability statistics. Behavior Research Methods \& Instrumentation, 14, 42 .

CoHEN, J. A. (1960). A coefficient of agreement for nominal scales. Educational \& Psychological Measurement, 20, 37-46.

FLEISS, J. L. (1981). Statistical methods for rates and proportions (2nd ed.). New York: Wiley. 
FLEISS, J. L., \& COHEN, J. (1973). The equivalence of weighted kappa and the intraclass correlation coefficient as measures of reliability. Educational \& Psychological Measurement, 33, 613-619.

Powers, S. (1985). A Pascal program that assesses the interrater reliability of nominal scales. Educational \& Psychological Measurement, 45, 613-614
WATKINS, M. W., \& LARIMER, L. D. (1980). Interrater agreement statistics with the microcomputer. Behavior Research Methods \& Instrumentation, 12, 466.

(Revision accepted for publication October 23, 1986.)

\title{
NOTICE
}

\author{
Abstracts of All Psychonomic Society Journal Articles Published \\ in the Bulletin of the Psychonomic Society
}

With the March 1987 issue, the Bulletin of the Psychonomic Society will launch the publication of a section that will contain abstracts of all articles that have appeared in recent issues of the other five Psychonomic Society journals-Animal Learning \& Behavior; Behavior Research Methods, Instruments, \& Computers; Memory \& Cognition; Perception \& Psychophysics; and Psychobiology. It is hoped that this feature will provide readers with additional access to information that will be of use in their work. 